\title{
Timelike Entanglement For Delayed-Choice Entanglement Swapping
}

\author{
David Glick \\ Department of Philosophy \\ University of Sydney
}

\begin{abstract}
Experiments involving delayed-choice entanglement swapping seem to suggest that particles can become entangled after they've already been detected. This astonishing result is taken by some to undermine realism about entanglement. In this paper, I argue that one can offer a fully realist explanation of delayed-choice entanglement swapping by countenancing timelike entanglement relations. I argue that such an explanation - radical though it may be -isn't incoherent and doesn't invite paradox. I compare this approach to the antirealist alternative and a more deflationary realist strategy defended by Egg (2013), each of which face certain challenges. The upshot is that we should take seriously the possibility of timelike entanglement and seek to develop a framework for quantum theory which allows for it.
\end{abstract}

\section{The Puzzle: Delayed-Choice Entanglement Swapping}

Entanglement swapping is a procedure in which entanglement may be "swapped" from a pair of jointly measured particles to a pair of particles lacking common prepartion. The technique has become quite commonplace in experiments involving entanglement and has numerous applications in quantum information theory (Bouwmeester et al., 2000). A simple experimental arrangement is depicted below (figure 1). Consider two sources that each produce a pair of photons in the state $\left|\psi^{-}\right\rangle=\frac{1}{\sqrt{ } 2}(|H V\rangle-|V H\rangle)$. One source produces the entangled pair $(1,2)$ and the other produces $(3,4)$. Initially, the quantum state of the fourparticle system is simply the product of two pair states $|\Psi\rangle=\left|\psi^{-}\right\rangle_{12} \otimes\left|\psi^{-}\right\rangle_{34}$. This state is separable into the states $\left|\psi^{-}\right\rangle_{12}$ and $\left|\psi^{-}\right\rangle_{34}$, each of which is an entangled two-photon state. Accordingly, there are two distinct entanglement relations $-R_{12}$ and $R_{34}$ - but no such relations between the pairs or between photons from different pairs.

The outer particles are sent off to polarization detectors at Alice and Bob while the inner particles are sent to a common location, Victor, which contains a switchable Bell-state analyzer. When switched on, a Bell-state measurement (BSM) is performed, which has the effect of projecting the incident particles 


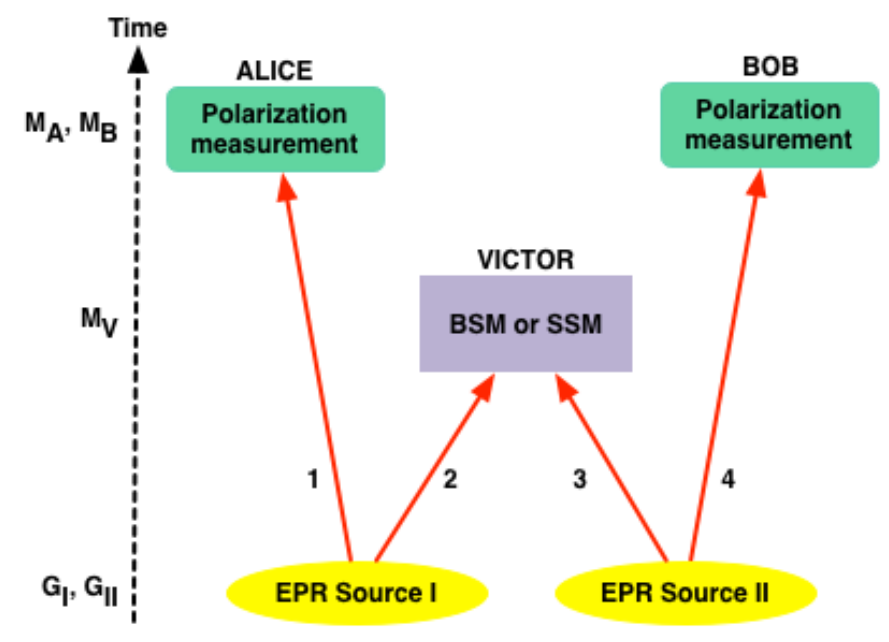

Figure 1: Entanglement Swapping Configuration

into one of the four maximally-entangled Bell-states:

$$
\left|\psi^{ \pm}\right\rangle=\frac{1}{\sqrt{ } 2}[|H\rangle|V\rangle \pm|V\rangle|H\rangle], \quad\left|\phi^{ \pm}\right\rangle=\frac{1}{\sqrt{ } 2}[|H\rangle|H\rangle \pm|V\rangle|V\rangle] .
$$

Otherwise, a separable state measurement (SSM) is performed. If the analyzer is off and the particles are measured separately, then, as expected, Bell-type correlations are found between the pairs $(1,2)$ and $(3,4)$ as in an ordinary EPR experiment.

If the analyzer is on, however, particles 2 and 3 are projected into one of the entangled Bell-states and, as a result, the remaining particles 1 and 4 are projected into an entangled Bell-state as well. This can be seen by writing the initial four-particle state in the basis given by the Bell-states of $(2,3)$ :

$$
|\Psi\rangle=\frac{1}{2}\left[\left|\psi^{+}\right\rangle_{14}\left|\psi^{+}\right\rangle_{23}-\left|\psi^{-}\right\rangle_{14}\left|\psi^{-}\right\rangle_{23}-\left|\phi^{+}\right\rangle_{14}\left|\phi^{+}\right\rangle_{23}+\left|\phi^{-}\right\rangle_{14}\left|\phi^{-}\right\rangle_{23}\right]
$$

Given this expression of $|\Psi\rangle$, we can see that if a BSM is performed at Victor with a result of, say, $\left|\psi^{+}\right\rangle_{23}$, then the remaining particles are projected into the state $\left|\psi^{+}\right\rangle_{14}$, and similarly for the other Bell states. Crucially, regardless of the outcome of the BSM at Victor, photons 1 and 4 become entangled (and $(1,2)$, $(3,4)$ become disentangled) as a result. Thus, we may say that entanglement has been "swapped" from $(2,3)$ to $(1,4){ }^{1}$

\footnotetext{
${ }^{1}$ Taken at face value, this description assumes a collapse interpretation of quantum theory, which many reject. However, one could recover a similar description on non-collapse interpretations at the level of effective quantum states, i.e., one may suppose that Victor's BSM yields effective entanglement between $(2,3)$ and suitably decoheres $(1,2)$ and $(3,4)$ so as to render them effectively disentangled.
} 
Entanglement swapping has some important implications for the understanding of entanglement. First, the particles that become entangled have never previously interacted, even in their preparation. This means that entanglement cannot be understood as essentially the result of systems "[entering] into temporary physical interaction due to known forces between them, and when after a time of mutual influence the systems separate again, then they can no longer be described in the same way as before, viz. by endowing each of them with a representative of its own" (Schrödinger, 1935). Second, if one thinks that entanglement relations involve action at a distance, then action at a distance is capable of influencing relational dispositions in addition to monadic ones (cf., Berkovitz (2016)). In particular, on such a view, extant entanglement relations (between $(1,2)$ and $(3,4)$ ) may be ultilized to create new entanglement relations (between $(1,4)$ ). Yet, while the implications of entanglement swapping are significant, few have taken the phenomenon to present a major threat to realism about entanglement or the quantum state more generally ${ }^{2}$

Delayed-choice entanglement swapping, by contrast, seems to present a real puzzle for realist conceptions of entanglement. The procedure was proposed as a thought experiment by Peres (2000), but has now been realized experimentally by Ma et al. (2012) and others. We begin with two entangled systems as in the ordinary case, but rather than have Victor preform his measurement prior to Alice and Bob, we delay particles 2 and 3 so that Victor can perform his measurement after his colleagues. Recall that the argument given above - the transition of equation 2 to a state in which $(2,3)$ and $(1,4)$ are each in entangled Bell states - didn't specify any times. This suggests that we should expect the same result as in the ordinary swapping case. In particular, when Victor successfully performs a BSM, entanglement will be swapped to $(1,4)$.

And, in fact, these results seem to have been confirmed by an experiment conducted by Ma et al. (2012) depicted below (Figure 2). We begin as before: two pairs of entangled photons $(1,2)$ and $(3,4)$ are produced by two EPR sources in the state $\left|\psi^{-}\right\rangle_{12} \otimes\left|\psi^{-}\right\rangle_{34}$. At this point the photons 1 and 2 are mutually entangled, as are 3 and 4 , but the 4-particle state is separable, and hence there is no entanglement across the two pairs. Alice and Bob each perform a polarization measurement of their photons ( 1 and 4 , respectively) along one of three freelychosen axes $(|H\rangle /|V\rangle,|R\rangle /|L\rangle,|+\rangle /|-\rangle)$ and the data from these measurements are saved for later analysis. Particles 2 and 3, meanwhile, enter an optical delay and only reach Victor at time $M_{V}$, nearly 500ns after $M_{A}$ and $M_{B}$, the times at which Alice and Bob perform their measurements.

As before, Victor "chooses" between performing a Bell-state measurement (BSM) or a separable state measurement (SSM) on $(2,3)$. In the actual experiment, the switchable Bell-state analyzer was linked to a quantum random number generator which determined the measurement (BSM or SSM) to be performed. The photons 2 and 3 are projected into either an entangled state $\left(\left|\phi^{+}\right\rangle_{23}\right.$ or $\left.\left|\phi^{-}\right\rangle_{23}\right)$ if a BSM is performed or a separable state in the case of a

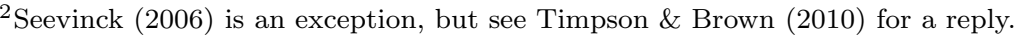




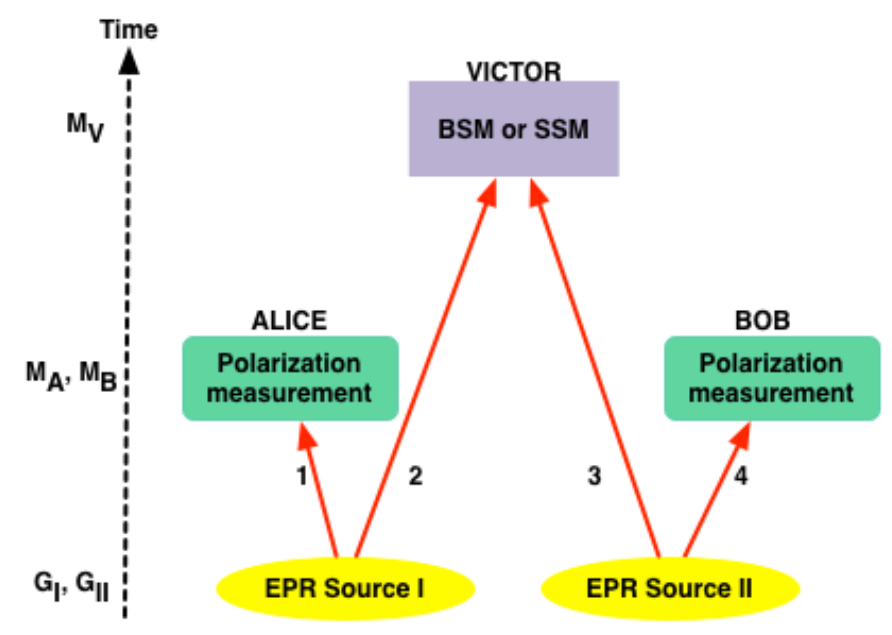

Figure 2: Delayed-choice entanglement swapping arrangement

SSM. When Victor's results are compared with those of Alice and Bob, they are found to be consistent with ascribing an entangled state to photons 1 and $4\left(\left|\phi^{+}\right\rangle_{14}\right.$ or $\left.\left|\phi^{-}\right\rangle_{14}\right)$ when a BSM is performed and a separable state otherwise. Thus, it seems that entanglement has been swapped to particles $(1,4)$ after they have already been detected (at $\left.M_{V}\right)$ !

This presents the following challenge: In the ordinary entanglement swapping case, Victor has the power to entangle (or not) the outer particles $(1,4)$ at a distance. In the delayed-choice case, it seems that Victor has the same power to entangle $(1,4)$. However, at the time of Victor's measurement (or choice), $(1,4)$ have already been detected. Thus, Victor's measurement must not only be capable of influence at a (spacelike) distance, but also backwards in time. The only way Victor can entangle $(1,4)$ is to act on them retrocausally when (or before) they are measured $\left(t \leq M_{A}, M_{B}\right)$. Moreover, if Victor's ability is grounded in extant entanglement relations, it seems that these must be relations of entanglement across time ${ }^{3}$

\section{Timelike Entanglement}

Several authors have suggested that delayed-choice entanglement swapping (DCES) undermines realism about entanglement. Here, for instance, is Peres's assessment in his original discussion of DCES:

\footnotetext{
${ }^{3}$ Writing in Aeon magazine, Elise Crull (2018) claims that a variant of the delayed-choice entanglement swapping experiment performed by (Megidish et al. 2012) reveals "temporal nonlocality," which is closely related to timelike entanglement. I'll have a bit more to say about temporal nonlocality in section 6 below.
} 
...if we attempt to attribute an objective meaning to the quantum state of a single system, curious paradoxes appear: quantum effects mimic not only instantaneous action-at-a-distance but also, as seen here, influence of future actions on past events, even after these events have been irrevocably recorded. (Peres, 2000, 141)

Ma et al. draw a similar conclusion after arguing that their results support the existence of DCES:

If one views the quantum state as a real physical object, one could get the seemingly paradoxical situation that future actions appear as having an influence on past and already irrevocably recorded events. However, there is never a paradox if the quantum state is viewed as to be no more than a "catalogue of our knowledge." (Ma et al. $2012,483)$

Finally, Richard Healey takes DCES to lend support to his pragmatist interpretation of quantum theory by undermining entanglement realism:

The delayed-choice entanglement-swapping experiment reinforces the lesson that quantum states are neither descriptions nor representations of physical reality. In particular, it undermines the idea that ascribing an entangled state to quantum systems is a way of representing some new, non-classical, physical relation between them. (Healey, 2012, 31)

In section 3 below, I will consider the alternative, anti-realist account of DCES these authors prefer. But, here I wish to claim that there is no obvious paradox or contradiction in a fully realist interpretation of DCES. Moreover, by positing timelike entanglement, one can provide the same form of explanation in all instances of entanglement swapping.

To begin, recall the ordinary swapping case. The realist explanation of Belltype correlations between Alice and Bob's measurement outcomes follows from these key claims:

1. Initially, there is entanglement between particles $(1,2)$ and $(3,4)$.

2. When Victor performs a BSM on particles $(2,3)$, he thereby creates an entanglement relation between them.

3. Victor's measurement also has the effect of entangling particles $(1,4)$.

4. The entanglement of $(1,4)$ explains the violation of a Bell inequality in Alice and Bob's measurement results.

Note that (1) is what allows for the move from (2) to (3); the reason Victor can entangle $(1,4)$ remotely is because there is extant entanglement relating each of them to particles 2 and 3, with which he can directly interact. In this way, entanglement mediates the influence Victor is able to exert on $(1,4)$. Then, once we have an entanglement relation between $(1,4)$, it may enter into our explanation 
of Bell-type correlations in the standard way; in some sense, the correlations in measurement results are the effect of a physical entanglement relation between the particles measured. The details of this explanation will depend on the particular interpretation of quantum theory in general (and quantum measurement in particular) one favors.

What would this explanation look like applied to DCES? Exactly the same! If Victor's BSM on $(2,3)$ is able to entangle $(1,4)$, then there must be extant entanglement relating $(1,2)$ and $(3,4)$. But, of course, $(1,4)$ have already been measured in an DCES experiment, so we must suppose that there are timelike entanglement relations, namely, those between (a) particle 1 at $t \leq M_{A}$ and particle 2 at $M_{V}$ and (b) particle 3 at $M_{V}$ and particle 4 at $t \leq M_{B}$. If we can make sense of such timelike entanglement relations, we can extend the explanation of swapping to the case of DCES. 4

But is timelike entanglement a coherent notion? Or is there something about the realist understanding of entanglement relations that prevents their extension into the temporal dimension? Of course, it's difficult to say anything significant about nature of entanglement outside the context of a (particular version of a) particular interpretation of quantum theory. For our purposes here, it will suffice to consider two broad families of thinking about entanglement that cross-cut interpretations qua solutions to the measurement problem. This taxonomy is not exhaustive, but captures a number of approaches to entanglement in versions of (e.g.,) Bohmian mechanics, dynamical collapse theories, modal interpretations, and "textbook" quantum mechanics. Thus, if both families are consistent with timelike entanglement, that goes some way toward establishing the conceptual coherence of the notion.

Action at a Distance (AAD): entangled particles are capable of unmediated non-local influence.

Non-separability (NS): entangled particles are fundamentally not wholly ontologically distinct.

There is much to be said about each strategy and how they relate to the various realist interpretations (for a start, see Healey (2016); Berkovitz (2016).) My claim is simply that on either understanding, timelike entanglement is novel, but not inconsistent. This shows that, at least for advocates of either approach, there is no paradox involved in the fully realist analysis of DCES suggested above.

\footnotetext{
${ }^{4}$ What are the relata of such entanglement relations? Here I follow standard practice in speaking of entanglement between particles, but more carefully, entanglement relations hold between states of particles which are indexed to a particular time. Hence, in the $\psi^{-}$state, the polarization state of each particle is entangled. Timelike entanglement would retain these time-indexed states as the relata of entanglement relations but allow that they be indexed to different times. As discussed in section 5 below, this may require revising the standard quantum mechanical formalism.
} 


\subsection{Timelike $A A D$}

Taken at face value, DCES seems to suggest retrocausation for the AAD approach. Victor's measurement seems to cause particles $(1,4)$ to become entangled at, or prior to, the time at which they are detected by Alice and Bob. If this supposition is correct, and retrocausation itself is incoherent, then timelike AAD does indeed lead to paradox in the case of DCES. However, there are good reasons to reject each step of this argument.

First, the supposition that there is retrocausality involved may be resisted. For instance, one may claim that the earlier entanglement of $(1,4)$ at $M_{A}, M_{B}$ causes Victor's choice to perform a BSM at $M_{V}$. This would seem to have problematic implications for Victor's free will-suggesting that his choice to perform a BSM wasn't truly free-but such considerations aren't necessary decisive 5 After all, as noted by Evans et al. (2012, 317), the popular "block universe" understanding of time has it that there are facts about the future actions of apparently free agents. Of course, whether the block universe is compatible with free will is a controversial matter, but the point is simply that one doesn't dismiss special relativity on the grounds that it rules out genuine free will. Similarly, the suggestion that Alice and Bob's measurements fix a fact about the future choice of a free agent doesn't amount to an obvious contradiction.

A quite different way to reject retrocausality is to deny that the AAD involved in entanglement is causal. There is a long history of attempts to reconcile $\mathrm{AAD}$ with special relativity that seek to diminish the causality of the influence in question. In this context, Abner Shimony coined the memorable phrase "passion at a distance" for a non-causal influence involved in quantum non-locality. Moreover, the no-signalling theorems of (e.g.,) Ghirardi et al. (1980); Jordan (1983); Redhead (1987) establish that no local measurement will allow for superluminal communication via a shared pair of entangled particles. This might further motivate the sense that the superluminal influence involved in AAD isn't properly understood as causal ${ }^{6}$ If entanglement-related AAD isn't causal, then timelike entanglement doesn't imply retrocausation.

Second, even if we grant that retrocausation is involved, there is no reason to think that this is untenable. While there may be some accounts of causation that preclude retrocausation, many accept at least its conceptual possibility. There are, of course, well-known paradoxes associated with retrocausation - the grandfather paradox and its ilk-but there is reason to doubt their relevance here. As noted above, the no-signalling theorems severely constrain the uses of AAD, should it exist. For instance, one cannot use entanglement-related influence to send signals, or transfer energy or some other conserved quantity.

\footnotetext{
${ }^{5}$ As noted above, in the experiment of Ma et al. (2012), the "choice" to perform a BSM was made by a random number generator. Thus, the worry there would be whether the device was truly random, but the considerations on both sides would be similar.

${ }^{6}$ On the popular interventionist approach to causation, for instance, a necessary condition on a variable being a cause is that certain inventions on it reliably lead to changes in the effect (Woodward 2005). But, the impossibility of signalling suggests that the relevant interventions aren't possible in quantum theory, and hence, that this condition isn't met in the case of AAD.
} 
It's hard to see how one could generate an instance of the grandfather paradox without relying on a variety of retrocausation of a stronger form than the nosignalling theorems will permit.

If, however, it were possible to generate a paradox using entanglementrelated retrocausation, then $\mathrm{AAD}$ would already be in trouble in the ordinary spacelike case. Causal paradoxes result when we have inconsistent events in a causal loop (e.g., killing one's grandfather and being born). But now suppose that we have a causal loop involving spacelike events $A$ and $B$ (say, Alice and Bob's measurement results, respectively). One could then introduce an inconsistency in the loop, for example: $A$ causes $B$ and $B$ causes $\neg A$, resulting in a paradox. The upshot is that AAD advocates must place certain restrictions on entanglement-related causation for independent reasons, and these restrictions ensure that retrocausality will not lead to paradoxes (cf., Price \& Wharton (2015)).

\subsection{Timelike $N S$}

The NS strategy seeks to understand entanglement by treating entangled particles as something like "fragments of a more fundamental whole." There are several ways of cashing out such a metaphor. Entangled particles may be viewed as: aspects of an extended/multiply-located/scattered simple, parts that are less fundamental than the whole they compose, low-dimensional projections of single object located in a high-dimensional space, etc.. What these strategies all have in common is a rejection of the fundamental distinctness of entangled particles. This enables the NS proponent to give a "common ground" explanation of non-local behavior along the lines of common cause explanation, but based in non-causal grounding relations (Ismael \& Schaffer, 2016). In a word, spacelike correlations are unsurprising if they concern measurements of the same thing.

Our concern here is not with the details of versions of NS, but rather, whether it can sustain timelike entanglement. And there answer seems to be clearly, yes, it can. First, note that objects can be extended discontinuously (or scattered, multiply-located, etc.) in time as well as in space. A single performance of a play, for instance, exists both before and after intermission, but not in between. Second, on Schaffer's (2010) view, entanglement motivates not just holism, but priority monism, in which there is a single fundamental object - the Cosmosin which everything is grounded. But surely the Cosmos is unlimited in both spatial and temporal extent. Third, if NS is meant to apply to real experimental implementations of EPR scenarios, then some measure of temporal extension is needed. In any actual EPR experiment, Alice's and Bob's measurements will often be non-simultaneous (either because there is no preferred foliation, or because they fail to be perfectly simultaneous on the preferred foliation), so if the explanation of the correlations in their measurement data proceeds via NS, it seems that the fundamental whole must include both Alice's particle at $M_{A}$ and Bob's particle at $M_{B}$, where $M_{A} \neq M_{B}$.

DCES does, however, have important implications for the NS strategy. The natural way to understand NS on a collapse interpretation involves thinking of 
an entangled system $(1,2)$ as being disentangled (and the corresponding whole thereby destroyed) upon measurement of either of the particles it contains (1 or 2). However, if NS is to account for DCES, then this cannot occur. It is essential that the entangled pairs $(1,2)$ and $(3,4)$ are wholly present when Victor performs his BSM at $M_{V}>M_{A}, M_{B}$. This would suggest a tension with the NS strategy and collapse interpretations, which would seem to imply that Alice and Bob measurements disentangle (and thereby destroy) the wholes before Victor performs his measurement. Thus, it seems that NS cannot account for DCES if combined with a collapse interpretation of this form 7

Thus, it seems that for these two approaches, timelike entanglement implies a revision rather than a rejection of entanglement realism. Of course, challenges remain for making sense of timelike entanglement, but these have more to do with the details of AAD and NS rather than timelike entanglement as such. For example, one might worry that the application of timelike entanglement to this case makes entanglement trivial 8 In order to explain Bell-type correlations in the measurement results of Alice and Bob, the proponent of timelike entanglement claims that there is an entanglement relation between $(1,4)$ at the time of Alice's and Bob's measurement due to Victor's later BSM. This is motivated by the idea that equation 2 doesn't depend on the time-order of the measurements. However, that means that we could also argue that $(2,3)$ are entangled before Victor's BSM via the same equation. But then it would seem to follow that any particles could be considered entangled because they could be subject to a BSM in the future. This would render entanglement relations trivial, and hence, incapable of explaining Bell-type correlations.

As noted above, it's open to the advocate of timelike AAD to suppose that the earlier entanglement of $(1,4)$ causes the later BSM and entanglement of $(2,3)$. But, a more plausible approach would be to claim that while equation 2 is neutral with respect to the dependencies involved, there is reason to think Victor's BSM is responsible for the entanglement of $(1,4)$. On this view, $(1,4)$ are entangled at $M_{A}, M_{B}$ in virtue of Victor's later measurement (at $M_{V}$ ). Generalizing, a pair of particles in a DCES experiment are entangled only if there actually is a BSM performed in the future that swaps entanglement to them. Of course, one may not know whether such a measurement will be performed, and hence, may wish to leave it open that the particles one encounters may be entangled. But, this doesn't trivialize entanglement as it still only applies to certain pairs of particles, namely, those prepared in an entangled state or entangled via swapping. Such an account may be developed in either realist approach discussed. On AAD, Victor's measurement has a retrocausal (or noncausal influence) on the pair of particles $(1,4)$ at $t \leq M_{A}, M_{B}$. On NS, Victor's measurement gives rise to the $(1,4)$ whole that Alice and Bob both measure.

\footnotetext{
${ }^{7}$ Indeed, there are difficult questions involving measurement and dynamical evolution on any version of NS. When exactly do the entangled wholes in question exist and how are they created and destroyed? These challenges are compounded by the requirement of Lorentz invariance in special relativity (cf., Myrvold (2002a)).

${ }^{8}$ Thanks to an anonymous referee for raising this worry.
} 
There are difficulties in working out the details and timing of such processes but, I submit, these difficulties are by in large the same as those already faced by these approaches in the context of spacelike entanglement.

\section{Alternative 1: Anti-realism}

I've argued that one can allow for timelike entanglement without inviting paradox, but one may still feel that the revisionary metaphysics required to do so motivates consideration of a more deflationary approach. For those who adopt an epistemic (or non-ontic) understanding of the quantum state, ascribing a quantum state to a compound system never merits positing a physical entanglement relation among the subsystems. Rather, the quantum state functions as a "catalogue of our knowledge" useful for making predictions about future measurement outcomes, but not descriptive of reality in any straightforward manner.

On such a view, DCES presents no puzzles (apparent or otherwise). Alice, Bob, and Victor each have their own quantum state assignments. Based on the initial preparation, they may all start off ascribing the quantum state $|\Psi\rangle=$ $\left|\psi^{-}\right\rangle_{12} \otimes\left|\psi^{-}\right\rangle_{34}$. Alice, upon making her measurement of particle 1, updates her quantum state for the entire 4-particle system on the basis of her measurement result in accordance with Lüder's rule, and likewise for Bob. Victor, assuming he doesn't (or cannot) know Alice and Bob's results, also begins with $|\Psi\rangle$ and then updates accordingly. Thus, if Victor performs a BSM, then he will assign to particles $(1,4)$ an entangled state, but this means only that he will expect to find measurement results consistent with this state when he learns of Alice and Bob's results. Crucially, Victor's ascribing an entangled state to particles $(1,4)$ doesn't imply any physical entanglement relation between them at $M_{A}, M_{B}$ (or any other time), but rather, merely guides his credences about the measurement results obtained by Alice and Bob.

This is a coherent picture, and it succeeds in avoiding revisionary metaphysics, but it does so at a cost. In particular, it inherits the traditional problems of antirealisms: a lack of explanatory power and a failure to take the theory at face value. First, if one abandons the representational role of the quantum state, then they cannot provide explanations of phenomena in terms of the world it describes. In the case of entanglement, non-ontic views are incapable of explaining non-local phenomena (i.e., measurement results that violate a Bell inequality) by appeal to a physical entanglement relation. Of course, certain explanations are still available: one can note that the initial quantum state $|\Psi\rangle$ predicts certain non-local phenomena, and hence, one should expect to find them. But such explanations are little comfort for those who seek to know what it is about the world that accounts for the Bell-type correlations in Alice and Bob's measurement results. Second, there is a general worry that this story is a poor fit with what quantum theory seems to be telling us. After all, nearly all mature physical theories use mathematical objects to represent states - conditions of a physical object - and apply a dynamical evolution to these states to generate 
empirical predictions. When these predictions are borne out, it is extremely natural to regard the states and dynamics involved as descriptive of an underlying reality.

As noted, these are standard problems for antirealisms in a variety of contexts and are unlikely to move either advocates or critics of this alternative. I mention them here to emphasize the following: DCES changes little with respect to the considerations for or against realism about entanglement. If one is weary of revisionary metaphysics, then they will have given up on physical entanglement well before consideration of these experiments. And if one is happy to take metaphysical lessons from quantum theory, then DCES is a source of new insights rather than a paradox in need of resolution. As I've sketched in section 2, it is unlikely that DCES will result in an outright contradiction for any worked-out understanding of entanglement. In sum, if one is willing and able to incorporate spacelike entanglement into their picture of reality, there is no clear reason why timelike entanglement should lead them to abandon the realist endeavor.

\section{Alternative 2: Limited Realism}

An alternative interpretation of DCES is given by Egg (2013). Egg endeavours to provide a principled basis to accept the realist account of ordinary entanglement swapping but reject its extension to DCES. Egg's reply focuses on an aspect of Ma et al.'s DCES experiment that was omitted from the initial presentation. Unlike a simple EPR experiment, the correlations in the data recorded by Alice and Bob are only apparent once that data has been sorted into subensembles according to the measurement performed and results obtained by Victor. Once we sort the results obtained by Alice and Bob in this way, we find that the subsets of data associated with Victor performing a BSM exhibit correlations that violate a Bell inequality. This leads Egg to conclude the following:

The Bell measurement on the $[2,3]$ pair allows us to sort the $[1,4]$ pairs into four subensembles corresponding to the four Bell states. Without delayed choice, this has physical significance, because each $[1,4]$ pair really is in such a state after the $[2,3]$ measurement. But if the $[1,4]$ measurements precede the $[2,3]$ measurement, the $[1,4]$ pair never is in any of these states. This is entirely compatible with the fact that evaluating the $[1,4]$ measurements within a certain subensemble shows Bell-type correlations. (Egg, 2013, 1133, original emphasis)

Egg's proposal is that we should posit physical entanglement between $(1,4)$ only when Victor's measurement occurs before Alice's and Bob's $\left(M_{V}<M_{A}, M_{B}\right)$. This allows one to preserve realism about entanglement (and an ontic view of the quantum state more generally) without having to adopt the revisionary metaphysics outlined in section 2. However, there are at least two problems with the 
proposal: (a) it's hard to reconcile with special relativity, (b) it leave us unable to explain the correlations in Alice and Bob's results in a DCES experiment.

First, as Healey (2017) notes, it's unclear how to apply Egg's account to cases of spacelike entanglement swapping-i.e., when $M_{V}$ is spacelike to $M_{A}, M_{B}$. Consideration of such cases isn't purely of philosophical interest either, as experiments such as Hensen et al. (2015) utilize spacelike swapping to prepare entangled particles. There are two main avenues of response: (a) posit a preferred foliation or (b) relativize to a frame. Egg seems to have some sympathies for (a) as he notes that "[s]ome of the most widely discussed realistic versions of quantum theory (e.g., Bohmian mechanics and the matter-density version of GRW) involve a commitment to a preferred foliation of spacetime. If these proposals are reasonable, then so is the assumption that there is a definite (although undetectable) temporal ordering between any two events" (Egg, 2013, 1130, fn.7). But, of course, not all realists are comfortable with such an assumption in clear tension with special relativity. If DCES compelled realists to adopt a preferred foliation, I suspect that many would defect to the antirealist camp. That leaves option (b), in which whether $(1,4)$ become entangled is a frame-dependent manner (cf., Myrvold (2002b)). This is also a difficult position for realists to adopt. It is standardly assumed that matters of (fundamental) ontology should be invariant under changes of reference frame, but this view would require one to hold both that (a) entanglement is physically real and (b) whether entanglement obtains is frame-dependent. Thus, adopting this version of Egg's proposal would lead to substantial challenges for the entanglement realist in the context of spacelike swapping.

Putting this to one side, there is further difficulty with Egg's limited realism. Just as the antirealist is unable to explain non-local phenomena, Egg's proposal leaves one unable to account for the Bell-type correlations between Alice and Bob's measurement results in a case of DCES. As Egg notes, it "entirely compatible" with a lack of genuine entanglement that measurement results involving $(1,4)$ are correlated within a subensemble, but that is not to say the correlation has been explained. After all, the correlations here are strong enough to violate a Bell inequality, which is not at all unsurprising even given sorting. At a minimum, Egg owes us an explanation of why we should expect to find Bell-type correlations in this case. Moreover, Egg cannot appeal to the simple explanation given in the ordinary swapping case based on equation 2 as that equation no longer applies at $M_{V}$ on his proposal. In fact, this proposal must account for why that explanation is inappropriate in the delayed-choice case despite the fact that it doesn't depend on the time-order of the measurements (which all commute with one another). Thus, on Egg's proposal, one can account for violations of Bell inequalities in DCES neither by appeal to a physical entanglement relation, nor by appeal to Lüder's updating applied to equation 2. This is perhaps unsurprising given that the position is antirealist about entanglement in the context of DCES, but it urges us to consider whether the costs of timelike entanglement warrant a retreat to a position that realists fight hard to resist in ordinary cases of entanglement. 


\section{Formalizing Timelike Entanglement}

I've argued that timelike entanglement allows for an explanatory and conceptually coherent interpretation of DCES. Given the costs associated with the alternatives, one may do well to take the implications of DCES at face value and seek to incorporate timelike entanglement into our understanding of the quantum world. There is, however, an important lacuna in the discussion thus far, namely: there seems to be no way to represent timelike entanglement in the standard formalism of non-relativistic quantum mechanics. In ordinary quantum mechanics, the state of system 1 at a time corresponds to a density operator $\rho_{1}$ in the Hilbert space $\mathcal{H}_{1}$ for the system. The state of a composite system is represented by a density operator $\rho_{12}$ in the tensor-project Hilbert space $\mathcal{H}_{12}=\mathcal{H}_{1} \otimes \mathcal{H}_{2}$. Again, it is assumed that $\rho_{12}$ corresponds to the joint state of $(1,2)$ at a single time (regardless of whether 1 and 2 are spatially separated or degrees of freedom of a single physical object). Thus, timelike entanglement appears to require some new way of composing composite states from the standard tensor-product formalism; one that will allow for trans-temporal composition.

Now, there have been some attempts to find a contruction for the representation of states across time. For example, Fitzsimons et al. (2015) aim to treat correlations in the results of measurements carried out at different times in the same manner as those between measurements performed at spacelike separation. They propose using a pseudo-density operator composed of the measurement results in question, which reduces to an ordinary density operator when the measurements are spacelike. Interestingly, the authors use this construction to identify correlations indicative of causal relations, not entanglement. But, as they note, their measure of "causality" is nearly identical to measures of entanglement, so the difference may not be so great 9

However, Horsman et al. (2017) prove a no-go theorem that rules out the possibility of quantum states across time -including the proposal of Fitzsimons et al.- given a number of physically plausible criteria. This would seem to show that "there is something fundamentally misguided in attempting to treat quantum systems over time in the same manner as composite systems at a single time" (Horsman et al. 2017, 12). Yet, as the authors themselves emphasize, their theorem is only as powerful as its axioms, and hence, one may alternatively conclude that representing states across time requires relaxing some of the standard assumptions about compound quantum states. Indeed, this seems to be precisely what we would expect in trying to develop a new conception of quantum states applicable to collections of systems at different times.

For our purposes, it suffices to point out that this is an area of on-going research. Clearly some revision to the usual quantum formalism is required to

\footnotetext{
9 Leifer \& Spekkens (2013) is another attempt. Their aim is to support the analogy with classical probability theory in which conditional probabilities may be defined for events bearing any spatiotemporal relation. If compound quantum states can been made similarly independent of time, then perhaps they are really just classical probability distributions after all, as the Bayesian approach to quantum theory claims.
} 
make sense timelike entanglement, but further work is needed to see the best way to proceed. My aim in this paper has been to provide some motivation for a notion of timelike entanglement in DCES experiments, however the best way to express it formally may be. If it were clear that there is no construction capable of representing timelike entanglement, then the point would be moot, but the no-go theorem of Horsman et al. (2017) fails to establish anything as strong as this.

\section{Conclusion}

I've argued that timelike entanglement-understood in a realist mannerprovides a clear understanding of DCES. Given the difficulty of characterizing timelike entanglement in the standard quantum formalism, this may recommend modifying quantum theory in some manner. A similar conclusion is reached by Emily Adlam in a recent paper (Adlam, 2018). Adlam challenges the assumption of temporal locality in quantum theory - that is, " the assumption that the probabilities attached to the outcomes of a measurement performed at a given time depend only on the state of the world at that time" Adlam, 2018 , 1). Among the motivations for Adlam's challenge is entanglement in time, which she discusses in the context of the so-called "temporal Bell inequalities." The best-known of these is the Leggett-Garg inequality (Leggett \& Garg, 1985), which follows from the assumption of "macrorealism" and is violated by quantum theory. However, despite its formal similarity to Bell's inequality, the assumptions required to derive the Leggett-Garg inequality are far more controversial than those assumed by Bell, making it unclear just what it shows (Timpson \& Maroney, 2013). Similar remarks apply to the inequality derived by Brukner et al. (2004), which purports to demonstrate entanglement in time, but requires a strong assumption about the nature of quantum measurements. However, there are still other variations of temporal Bell inequalities, and some of these may involve more plausible assumptions. If so, these results could provide additional motivation for entanglement across time from those discussed here.

Regardless of the fate of the temporal Bell inequality program, DCES provides another reason to take timelike entanglement, and hence temporal nonlocality, seriously. Indeed, one may share Adlam's ambition that timelike entanglement "may ultimately point us not just to a new interpretation of quantum mechanics but to a new theory altogether" (Adlam, 2018, 17).

\section{References}

Adlam, E. (2018). Spooky action at a temporal distance. Entropy, 20, 41.

Berkovitz, J. (2016). Action at a distance in quantum mechanics. In E. N. Zalta (Ed.), The Stanford Encyclopedia of Philosophy. (Spring 2016 ed.).

Bouwmeester, D., Ekert, A. K., Zeilinger, A. et al. (2000). The Physics of Quantum Information volume 38. Springer Berlin. 
Brukner, C., Taylor, S., Cheung, S., \& Vedral, V. (2004). Quantum entanglement in time. arXiv preprint quant-ph/0402127, .

Crull, E. (2018). You thought quantum mechanics was weird: check out entangled time. URL: https://aeon.co/ideas/ you-thought-quantum-mechanics-was-weird-check-out-entangled-time.

Egg, M. (2013). Delayed-choice experiments and the metaphysics of entanglement. Foundations of Physics, 43, 1124-1135. URL: http://dx.doi.org/ 10.1007/s10701-013-9734-4.

Evans, P. W., Price, H., \& Wharton, K. B. (2012). New slant on the EPR-Bell experiment. The British Journal for the Philosophy of Science, (p. axr052).

Fitzsimons, J. F., Jones, J. A., \& Vedral, V. (2015). Quantum correlations which imply causation. Scientific reports, 5 .

Ghirardi, G.-C., Rimini, A., \& Weber, T. (1980). A general argument against superluminal transmission through the quantum mechanical measurement process. Lettere Al Nuovo Cimento (1971-1985), 27, 293-298.

Healey, R. (2012). Quantum theory: A pragmatist approach. The British Journal for the Philosophy of Science, 63, 729-771.

Healey, R. (2016). Holism and nonseparability in physics. In E. N. Zalta (Ed.), The Stanford Encyclopedia of Philosophy. (Spring 2016 ed.). URL: https: //plato.stanford.edu/archives/spr2016/entries/physics-holism/.

Healey, R. (2017). Quantum states as objective informational bridges. Foundations of Physics, 47, 161-173.

Hensen, B., Bernien, H., Dreau, A. E., Reiserer, A., Kalb, N., Blok, M. S., Ruitenberg, J., Vermeulen, R. F. L., Schouten, R. N., Abellan, C., Amaya, W., Pruneri, V., Mitchell, M. W., Markham, M., Twitchen, D. J., Elkouss, D., Wehner, S., Taminiau, T. H., \& Hanson, R. (2015). Loophole-free Bell inequality violation using electron spins separated by 1.3 kilometres. Nature, 526, 682-686. URL: http://dx.doi.org/10.1038/nature15759.

Horsman, D., Heunen, C., Pusey, M. F., Barrett, J., \& Spekkens, R. W. (2017). Can a quantum state over time resemble a quantum state at a single time? Proceedings of the Royal Society A, 473.

Ismael, J., \& Schaffer, J. (2016). Quantum holism: Nonseparability as common ground. Synthese, (pp. 1-30).

Jordan, T. F. (1983). Quantum correlations do not transmit signals. Physics Letters A, 94, 264.

Leggett, A. J., \& Garg, A. (1985). Quantum mechanics versus macroscopic realism: Is the flux there when nobody looks? Physical Review Letters, 54, 857. 
Leifer, M. S., \& Spekkens, R. W. (2013). Towards a formulation of quantum theory as a causally neutral theory of bayesian inference. Phys. Rev. A, 88, 052130. URL: https://link.aps.org/doi/10.1103/PhysRevA.88.052130 doi:10.1103/PhysRevA.88.052130.

Ma, X.-s., Zotter, S., Kofler, J., Ursin, R., Jennewein, T., Brukner, Č., \& Zeilinger, A. (2012). Experimental delayed-choice entanglement swapping. Nature Physics, 8, 479-484.

Megidish, E., Halevy, A., Shacham, T., Dvir, T., Dovrat, L., \& Eisenberg, H. S. (2012). Entanglement between photons that never co-existed. In Frontiers in Optics. Optical Society of America.

Myrvold, W. C. (2002a). Modal interpretations and relativity. Foundations of Physics, 32, 1773-1784.

Myrvold, W. C. (2002b). On peaceful coexistence: is the collapse postulate incompatible with relativity? Studies in History and Philosophy of Science Part B: Studies in History and Philosophy of Modern Physics, 33, 435-466.

Peres, A. (2000). Delayed choice for entanglement swapping. Journal of Modern Optics, 47, 139-143.

Price, H., \& Wharton, K. (2015). Disentangling the quantum world. Entropy, $17,7752-7767$.

Redhead, M. (1987). Incompleteness, Nonlocality and Realism. Clarendon.

Schaffer, J. (2010). Monism: The priority of the whole. Philosophical Review, $119,31-76$.

Schrödinger, E. (1935). Discussion of probability relations between separated systems. In Mathematical Proceedings of the Cambridge Philosophical Society (pp. 555-563). Cambridge Univ Press volume 31.

Seevinck, M. (2006). The quantum world is not built up from correlations. Foundations of Physics, 36, 1573-1586.

Timpson, C., \& Maroney, O. (2013). Quantum vs. macro-realism: what does the Leggett-Garg inequality actually test? The British Journal for the Philosophy of Science, .

Timpson, C. G., \& Brown, H. R. (2010). Building with quantum correlations. Quantum Information Processing, 9, 307-320.

Woodward, J. (2005). Making things happen: A theory of causal explanation. Oxford University Press. 